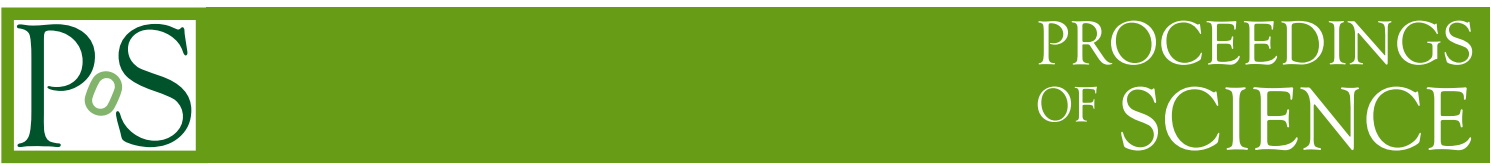

\title{
A search for aligned radio polarizations
}

\author{
Sanhita Joshi \\ Jodrell Bank Observatory, University of Manchester, Macclesfield, SK11 9DL \\ E-mail: sjoshiejb.man.ac.uk
}

\section{R. Battye, I.W.A. Browne, N. Jackson}

Jodrell Bank Observatory, University of Manchester, Macclesfield, SK11 9DL

\begin{abstract}
We have used the very large JVAS/CLASS $8.4-\mathrm{GHz}$ surveys of flat-spectrum radio sources to test the hypothesis that there is a systematic alignment of polarization position angle vectors on cosmological scales of the type claimed by Hutsemékers et al. (2005). The polarization position angles of 4290 sources with polarized flux density $\geq 1 \mathrm{mJy}$ have been examined. They do not reveal large-scale alignments either as a whole or when split in half into high-redshift (z $\geq 1.24$ ) and low-redshift sub-samples. Nor do the radio sources which lie in the specific areas covered by Hutsemékers et al. (2005) show any significant effect. We have also looked at the position angles of parsec-scale jets derived from VLBI observations and again find no evidence for systematic alignments. Finally, we have investigated the correlation between the polarization position angle and those of the parsec-scale jets. As expected, we find that there is a tendency for the polarization angles to be perpendicular to the jet angles. However, the difference in jet and polarization position angles does not show any systematic trend in different parts of the sky.
\end{abstract}

From Planets to Dark Energy: the Modern Radio Universe

October 1-5 2007

The University of Manchester, UK 


\section{Introduction}

In an isotropic universe the sizes of largest coherent structures are expected to be limited by the time taken for the primordial density fluctuations to undergo gravitational collapse. In practice both, observations and simulations, agree that the largest structures have scales $\leq 100 \mathrm{Mpc}$. So any observation of coherent structures on much larger scales than $100 \mathrm{Mpc}$ would have a profound implications on our understanding of the early universe.

Recently, Hutsemékers (1998); Hutsemékers and Lamy (2001); Hutsemékers et al. (2005) have been gathering evidence that that the linear polarization of quasars in optical wavelengths are nonuniformly distributed and are systematically different near North and South Galactic poles. The effect is more pronounced when the sample of 355 quasars is divided according to redshifts.

Stimulated by these hints we examined some linear polarization measurements made with the Very Large Array (VLA) between 1990 and 1999 of a large number of compact, flat-spectrum radio sources. Two surveys, JVAS (Patnaik et al., 1992; Browne et al., 1998; Wilkinson et al., 1998) and CLASS (Myers et al., 2003; Browne et al., 2003) were carried out, initially with different aims. But linear polarization data were also obtained at the same time for 16503 sources in all. Jackson et al. (2007) carefully reanalyzed the polarization data for this purpose. We made use of 4290 sources with polarized flux density $\geq 1 \mathrm{mJy}$ to search for possible polarization alignments.

\section{Statistical analysis}

A simple global test was designed and performed to see if the PAs are aligned, it is as follows: for every object, the $n$ nearest objects are checked for alignment. If the object has PA within $\Delta \theta$ of the object under consideration, it is considered aligned. The choice of the appropriate value of $n$ depends on the size of the sample, since the probability of finding $n$ alignments by chance should be low. It also depends upon the scale of real alignments which is, of course, not known. However, if the regions over which the alignments occur contain more than $n$ objects, the test will pick out many objects in the same area all of which have significantly more neighbours having aligned polarizations than expected by chance and real structures will not be missed. We initially chose $n=25$ and $\Delta \theta=45^{\circ}$ but other values for $n$ and $\Delta \theta$ were tried as well. The results of the test for $n=25$ and $\Delta \theta=45^{\circ}$ are shown in Fig. 1. The error bars have been derived from 5000 random realizations where, keeping positions of the objects the same, the sky was populated with sources having random PAs and the nearest neighbour test performed on each realization thus producing 5000 histograms like the one shown in Fig. 1. For each bin in the histograms the distribution of values was obtained and fitted by a Gaussian. The plotted points and error bars are, respectively, the means and standard deviations obtained from the Gaussian fits.

The same test was run on the optical data in region A1 defined by Hutsemékers et al. (2005) as $168^{\circ} \leq \alpha \leq 218^{\circ}$ and $\delta \leq 50^{\circ}$. The results for that are shown in Fig. 2. It is clear that, given the error bars, the real histogram is a reasonably good fit for the JVAS/ CLASS data, to that expected for a sample with randomly oriented PAs. Whereas, the optical data is far away from the random distribution. For this test, the degrees of freedom are not known so reduced $\chi^{2}$ cannot be calculated. So each random and the real distributions were compared with the average random distribution and $\chi^{2}$ was computed for each comparison. From the distribution of the random and real $\chi^{2}$ it was 
found that the probability of finding such a distribution by chance is 1 out of 2 for the radio data and 0.007 out of 1 for the optical data.

\section{Discussion and conclusion}

As it can be noted from the Fig. 1 that we do not find any alignments in the radio data. Out of 355 objects from the optical data $72 \%$ are radio loud, and optical and radio emission is predominantly synchrotron in origin. Thus for the majority of the radio and optical objects the radiation mechanism is the same, so an intrinsic effect can be ruled out.

Assuming the optical result is of cosmological significance, it can be argued that the Faraday rotation destroys alignments in radio. However, rotation measures would have to be of the order of thousands of radm ${ }^{-2}$ and this possibility can also be ruled out because (a) the rotation measures of such sources are often less then a few tens of radm $^{-2}$ (e.g. Rudnick and Taylor) and (b) the correlation between the position angles of radio jets and polarization PA is observed. Such a correlations would be destroyed if the polarization PA were significantly randomized by Faraday rotation.

We conclude that intrinsic mechanisms or Faraday rotation cannot acoount for the lack of radio alignments and therre the most viable explanations are that either there is some, as yet uncertain, propogation effect that works on the optical photons but not the radio ones, or the optical results have may not been interpreted correctly. This might be a result of small number statistics or a statistical fluke.

\section{References}

Browne I.W.A., Wilkinson P.N., Patnaik A.R., Wrobel J.M., 1998, MNRAS, 293, 257

Browne I.W.A., Wilkinson P.N., Jackson N.J.F., Myers S.T., Fassnacht C.D., Koopmans L.V.E., Marlow D.R., Norbury M., Rusin D., Sykes C.M., 2003. MNRAS 341, 13

Hutsemékers, D., Lamy, H., Sluse, D. 2005, A\&A, 441, 915

Hutsemékers, D., Lamy, H. 2001, A\&A, 367, 381

Hutsemékers D. 1998, A\&A, 332, 410

Jackson N., Battye R. A., Browne I. W. B., Joshi S., Muxlow T. W. B., Wilkinson P. N., 2007, MNRAS, 376, 371

Myers S.T., Jackson N.J., Browne I.W.A., de Bruyn A.G., Pearson T.J., Readhead A.C.S., Wilkinson P.N., Biggs A.D., Blandford R.D., Fassnacht C.D., 2003. MNRAS 341, 1

Patnaik, A. R., Browne, I. W. A., Wilkinson, P. N., Wrobel, J. M. 1992, MNRAS, 254, 655

Wilkinson, P. N., Browne, I. W. A., Patnaik, A. R., Wrobel, J. M., Sorathia, B., 1998, MNRAS, 300,790 

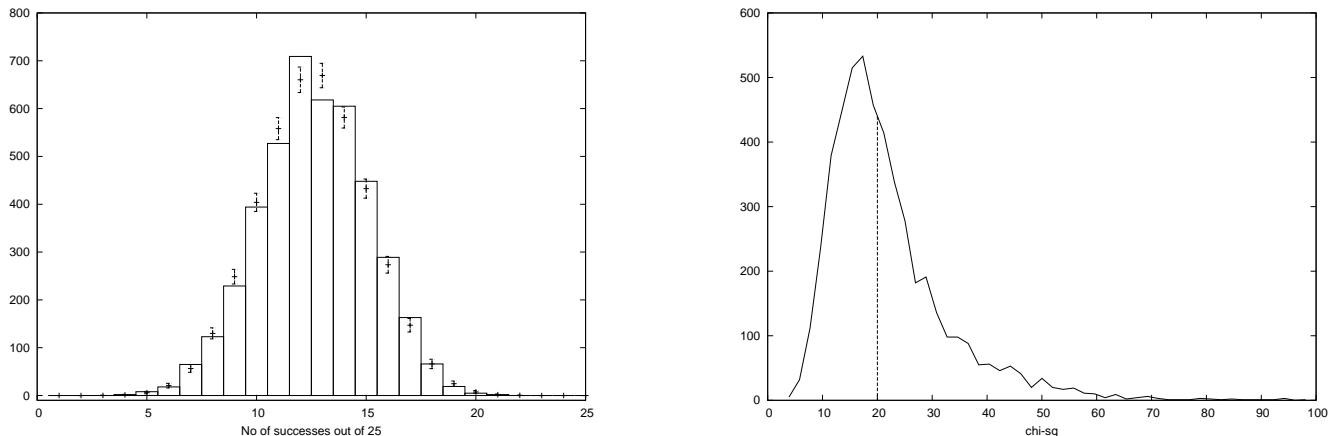

Figure 1: Comparison of nearest neighbour distribution for the 4290 JVAS/CLASS objects with that expected for a randomly oriented sample obtained from 5000 random realizations. The solid lined-histogram is for the real data and dashed data with error bars is from 5000 random samples. $\chi^{2}$ for this distribution is 20.1. The right hand panel shows the distribution of $\chi^{2}$ obtained by comparing each random realization with the averaged of all the random ones. The line represents $\chi^{2}$ for the observed sky.
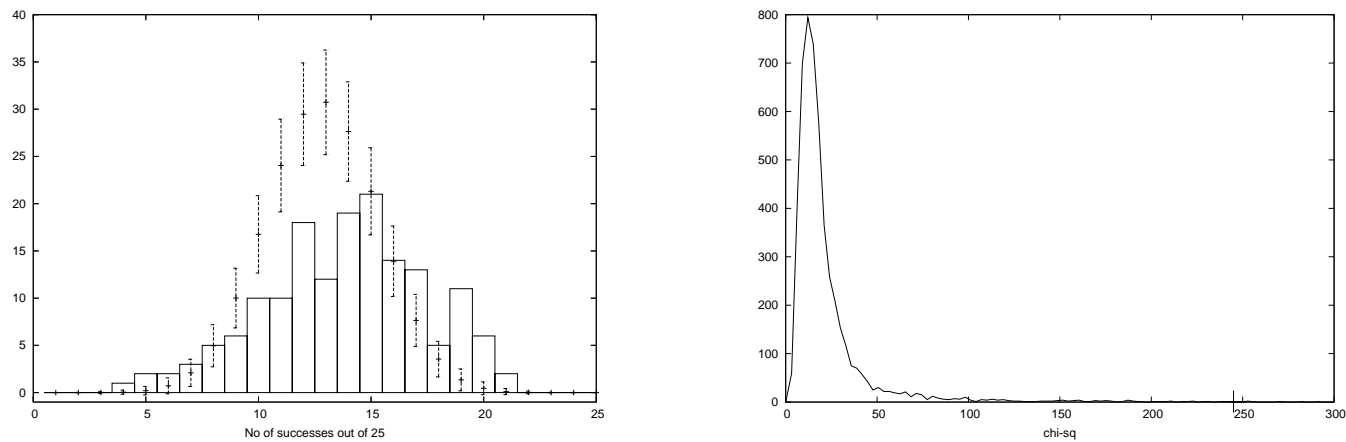

Figure 2: The panel on the left shows the histogram from nearest neighbour test for region A1 on the optical data from Hutsemékers et al. (2005), with error bars from 5000 random realizations. The panel on the right shows the $\chi^{2}$ distribution for random realizations with the line showing that for the real sky in region A1. For these $\chi^{2}$ is 245 with probability of $0.7 \%$ under the hypothesis that the distribution of position angles is uniform. 